\title{
A MCM-based Microsystem for Biological Fluids Analysis by Optical Absorption
}

\author{
G. Minas, J. C. Ribeiro, G. de Graaf, R. F. Wolffenbuttel and J. H. Correia \\ Dept. Industrial Electronics, University of Minho, Guimaraes, Portugal, gminas@dei.uminho.pt \\ Dept. Industrial Electronics, University of Minho, Guimaraes, Portugal, jcaribeiro@dei.uminho.pt \\ Fac. EEMCS, Microelectronics, Delft University of Technology, Delft, The Netherlands, g.degraaf@ewi.tudelft.nl \\ Fac. EEMCS, Microelectronics, Delft University of Technology, Delft, The Netherlands, r.f.wolffenbuttel@ewi.tudelft.nl \\ Dept. Industrial Electronics, University of Minho, Guimaraes, Portugal, Higino.Correia@dei.uminho.pt
}

\begin{abstract}
This paper presents a MCM (Multi-Chip-Module) microsystem for biological fluids analysis. It is composed of three parts: (1) a CMOS silicon optical detection microsystem which includes a photodetector and a light-tofrequency converter for readout; (2) a dielectric thin-film based optical filter on top of the photodetector fabricated using IC-compatible post-processing; (3) a glass die on top containing the microchannels fabricated using SU-8 techniques. The application is in the low-cost measurement of concentration of biomolecules in biological fluids, by the optical absorption in a part of the visible spectrum defined by the specific molecule. Signals proportional to the intensity of the light transmitted through the biological fluid are available at the output in the form of bit streams, which allows simple computer interfacing. The quantitative measurement of total protein in urine is successfully demonstrated. The photodiode responsivity is $224 \mathrm{~mA} / \mathrm{W}$ with a FWHM (Full-Width-Half-Maximum) of $10 \mathrm{~nm}$ at $\lambda=600 \mathrm{~nm}$. The optical system sensitivity is $1 \mathrm{kHz} / \mathrm{Wm}^{-2}$ at $\lambda=670 \mathrm{~nm}$.
\end{abstract}

\section{Keywords}

MCM (Multi-Chip-Module), SU-8 techniques, biomolecules analysis, optical absorption.

\section{INTRODUCTION}

For diagnostic reasons patients in a hospital are often subjected to biochemical analysis of their biological body fluids. Usually the analyses are carried out in clinical laboratories and the results become available after several hours, sometimes days. As a consequence a reliable diagnosis cannot be performed within the consultation time. Mistakes in the logistics, such as lost samples and mislabeling, may further delay diagnosis [1]. The automated equipment used in a state-of-the-art laboratory reduces errors, but use high sample and reagent volumes, making the analysis systems expensive and does not contribute to patient comfort [2]. Outside the laboratory environment, reagent strips are commercially available [3]. Such strips are available for a limited set of biomolecules to be analyzed and the color readout is merely qualitative. The need for rapid and on-line measurements with low sample volumes led to the development of microsystems with the fluidic, detection and readout systems integrated in a single module.

This paper describes a MCM-based microsystem for application in clinical analysis, especially in the spectrophotometric analysis of biological fluids. It allows the measurement of the concentration of biomolecules in those fluids. That measurement is based on colorimetric detection by the optical absorption in a part of the visible spectrum defined by the reaction of the specific molecule with a specific reagent. The microsystem combines in a MCM the detection and readout system, the optical filtering system and the microfluidic system.

\section{MICROSYSTEM DESIGN}

The optical detection microsystem avoids the need for expensive readout optics and may enable low-cost disposable devices, which would improve the use of spectrophotometric analysis in clinical diagnostics. The optical channel is composed of: the fluidic channel, a thin-films optical filter and a silicon photodetector (see Figure 1). The structure is optimized for optical response at the absorption peak of a particular molecule. The optical absorption intensity is proportional to the biomolecule concentration.

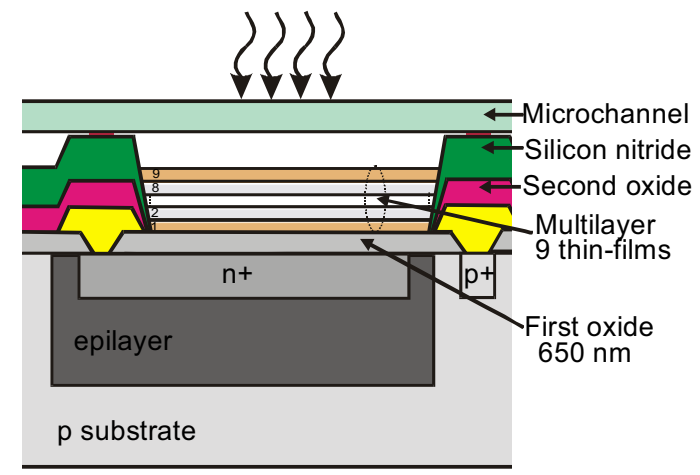

Figure 1. Cross-section of the designed CMOS compatible optical channel.

\section{Detection and Readout System}

The design involves layer selection for the photodetector and selection of the dielectric layers available in the CMOS standard process. The photodetector is a pn-junction photodiode fabricated using an $\mathrm{n}$-diffusion/p-substrate 
junction. This structure is chosen, because it provides the best possible quantum efficiency in the desired spectral range of photodiodes available in a CMOS process [4]. A light-to-frequency converter is integrated with the photodiodes to convert the photocurrent into a semi-digital signal (see Figure 2). Four photodiodes are used in each measurement. The first photodiode is for the reagent (without biomolecules), the second is for the biological fluid being analyzed, the third is for measuring the photodiode dark current and the fourth is for the calibrator, a standard with a well-known concentration of the biomolecule that is being analyzed.

The reverse biased junction capacitance of the photodiode $C_{j}$ and capacitor $C_{f b}$ are used as storage elements. The logic and the analog switches $S_{2 a}$ though $S_{2 d}$ select the channel to be measured. These switches are identical complementary PN-MOS switches. The comparator is a clocked highspeed regenerative comparator with a rail-to-rail input circuit. For reliable operation a two-phase non-overlapping clock is used for the analog switch $S_{1}$ and for the comparator. The frequency of the output converter is directly proportional to the input photocurrent [5] and hence to the biomolecules concentration, with $f_{\text {bistream }}=I_{s} /\left(V_{\text {ref }} \times C_{f b}\right)$.

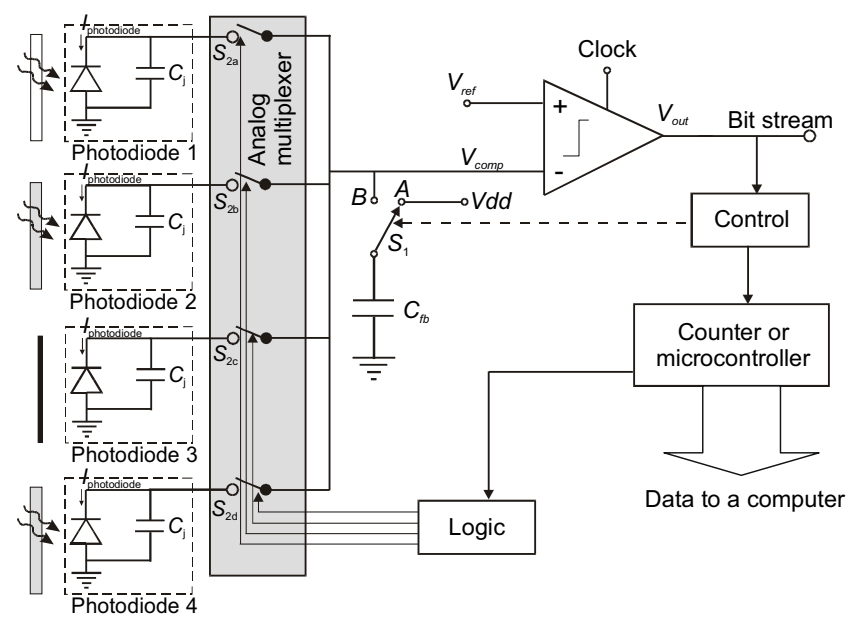

Figure 2. Block diagram of the photodiode readout circuit.

\section{Optical Filtering System}

An optical filter is required with a narrow passband around the wavelength for which the biomolecule being analyzed has its absorption maximum, is postprocessed on top of the photodiode. It allows that the photodiode measures the intensity of only the desired wavelength transmitted through the mixture. The optical filter is based on a 9 layer dielectric thin-film design, which offers high reflectivity with low absorption losses. The multilayer is structurally optimized together with the transmission through the three dielectric layers on top of the pn-junction provided by the CMOS process.

\section{Microfluidic System}

The microfluidic system contains the microchannels and the detection chambers (see Figure 3). There are three detection chambers, each $2 \mathrm{~mm}$ wide, $3 \mathrm{~mm}$ long and $500 \mu \mathrm{m}$ deep. The high depth is crucial for the optical absorption measurements. The detection chamber, $\mathbf{A}$, contains only the reagent and it is needed to obtain the baseline reference and to calibrate the light source. The detection chamber, $\mathbf{B}$, allows the mixed solution analysis; it contains the reagent plus the sample with biomolecules. The detection chamber, $\mathbf{C}$, is needed to calibrate the biomolecule concentration; it contains a well-known concentration standard of the biomolecule being analyzed.

The main channel is $500 \mu \mathrm{m}$ wide, $70 \mathrm{~mm}$ long and $500 \mu \mathrm{m}$ deep. It has two inlets, one for the reagent and the other for the sample. The chosen structure allows a complete and homogeneous mixing of the reagent with the sample [6].

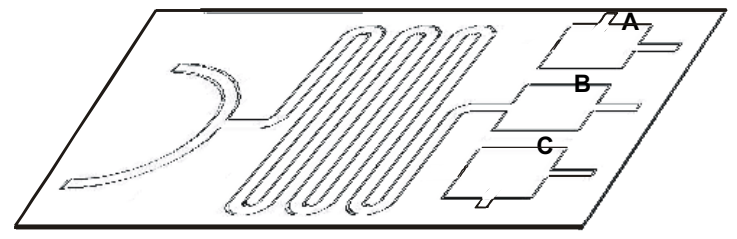

Figure 3. Layout of the microfluidic system.

\section{MICROSYSTEM FABRICATION}

\section{Detection and Readout System}

The CMOS compatible photodetectors and readout circuits have been fabricated through a double-metal, singlepolysilicon, $1.6 \mu \mathrm{m}$ n-well CMOS process. The area of each optical channel is $500 \times 500 \mu \mathrm{m}^{2}$. Figure 4 shows a photograph of the fabricated optical detection microsystem.

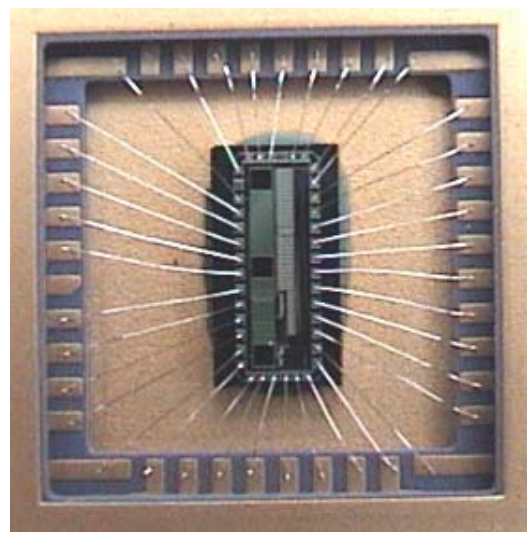

Figure 4. A photograph of the optical detection microsystem.

\section{Optical Filtering System}

The optical filter is composed of a multilayer stack of $\mathrm{TiO}_{2}$ and $\mathrm{SiO}_{2}$ thin-films. The thickness of the films determines the tuned wavelength. $\mathrm{SiO}_{2}$ has been selected because the dependence of its refractive index on the wavelength for 
the spectral band between $500 \mathrm{~nm}$ and $700 \mathrm{~nm}$ is almost constant (1.465 to 1.457 , respectively). $\mathrm{TiO}_{2}$ have been selected due to fabrication constraints (the deposition process is well-characterized).

The optical filters are postprocessed on top of the photodetector using the same mask that was used for the selection of the dielectric layers available in the CMOS standard process.

\section{Microfluidic System}

Figure 5 shows a photograph of the microchannels die fabricated using SU-8 techniques. The SU-8 fabrication process is a low-cost process, biocompatible, UV lithography semiconductor compatible and does not require expensive masks. Moreover, SU-8-based processing enables the fabrication of deep microchannels with very low sidewall roughness and is suitable for optical absorption measurement [7].

A negative mask of the microchannels die layout is fabricated from a regular transparency foil. The SU-8 photoresist chosen is the SU-8 100 (the best for deep channels). The microchannels die fabrication starts with the spinning of the SU-8 photoresist on a glass substrate at $700 \mathrm{rpm}$ during $100 \mathrm{~s}$. The pre-expose bake is performed by soft bake at $90{ }^{\circ} \mathrm{C}$ during $3000 \mathrm{~s}$, the second spun is done at $700 \mathrm{rpm}$ during $100 \mathrm{~s}$ and a soft bake during $300 \mathrm{~s}$ at $50{ }^{\circ} \mathrm{C}$ is again performed. The temperature is ramped up to $90{ }^{\circ} \mathrm{C}$, during $5400 \mathrm{~s}$, and subsequently ramped down again. The exposure is during $900 \mathrm{~s}$ on an Electronic Vision EV-420 mask and bonding aligner. Post-exposure bake is performed during $300 \mathrm{~s}$ at $50^{\circ} \mathrm{C}$. The temperature again is ramped up to $90^{\circ} \mathrm{C}$ during $1200 \mathrm{~s}$ and ramped down. The fabrication ends with the development during $2700 \mathrm{~s}$ in the SU-8 developer.

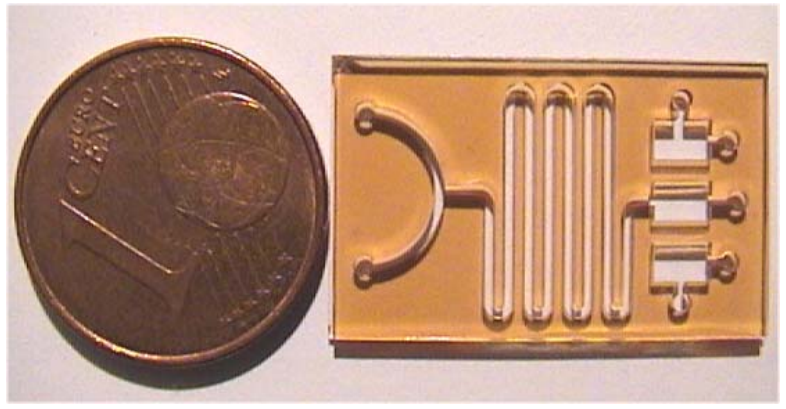

Figure 5. A photograph of the SU-8 based structure microchannels.

\section{EXPERIMENTAL RESULTS}

The MCM-based microsystem was tested in the measurement of the total protein concentration in a urine sample. The reagent used in the measurements was the Microprotein-PR reagent from Sigma-Aldrich. It reacts with a sample of urine containing total protein in a 50:1 ratio, and produces an absorption maximum at a specific wavelength $(\lambda=600 \mathrm{~nm})$.

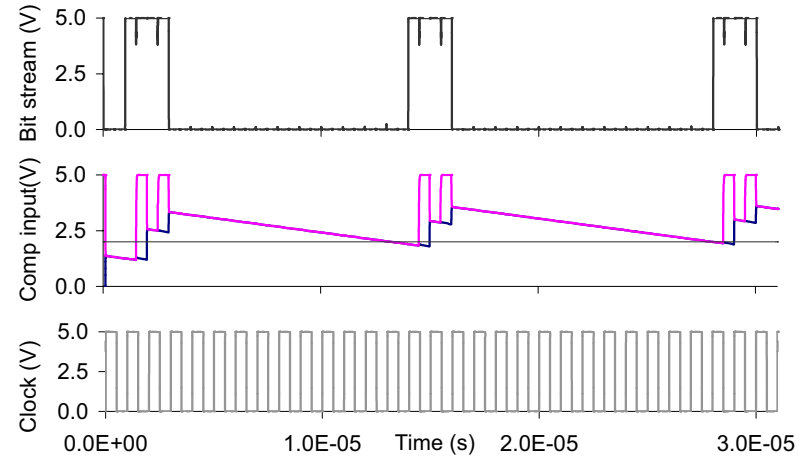

Figure 6. Characteristic signals in the readout circuits.

The photodiode current was measured using a Keithley 487 picoammeter. Figure 6 presents the bit stream output at a photodiode current of $137 \mathrm{nA}$, which represents $15 \mathrm{mg} / \mathrm{dl}$ of total protein concentration in urine. The photodiode dark current is $0.38 \mathrm{pA}$ at $0 \mathrm{~V}$ and the sensitivity achieved is 1 $\mathrm{kHz} / \mathrm{Wm}^{-2}$ at $\lambda=670 \mathrm{~nm}$ (using the TLS230 from Texas Instruments as reference).

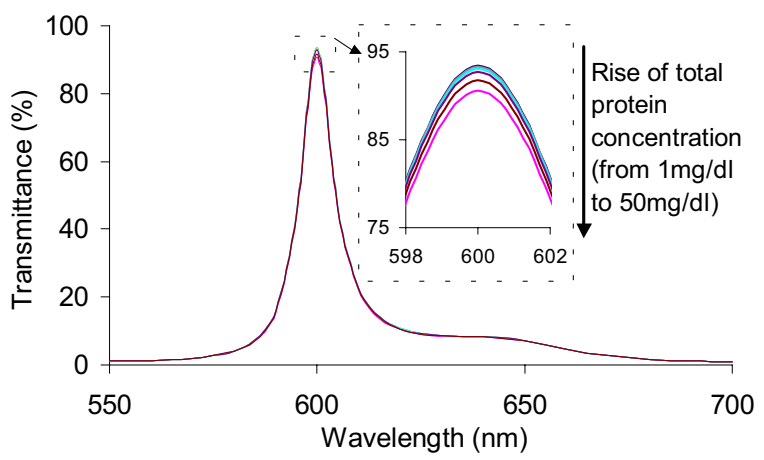

Figure 7. Measured transmittance spectra for different total protein concentration (optical effect of photodiode and interference filter included).

Figure 7 shows the measured transmittance through the entire optical channel as a function of protein concentration. A $250 \mathrm{~W}$ quartz/tungsten/halogen lamp with a monochromator ORIEL Cornerstone $130^{\mathrm{TM}}$ was used as light source. Measurement results were done from $1 \mathrm{mg} / \mathrm{dl}$ to $50 \mathrm{mg} / \mathrm{dl}$, comprising the range of normal and usually abnormal values in a human being (normal values are less than $20 \mathrm{mg} / \mathrm{dl}$ ). These measurements show that the fabricated optical channel is sensitive to a narrow spectral band centered at the wavelength for which the biomolecule being analyzed has its absorption maximum $(\lambda=600 \mathrm{~nm})$, with a $\mathrm{FWHM}=10 \mathrm{~nm}$.

\section{CONCLUSIONS}

The reported MCM microsystem offers a new approach for clinical analysis, especially in biological fluid analysis, due to its portability ensuring that the analysis can be performed at any location with instantaneous results. Moreover, its optical detection and filtering system avoids the need of expensive readout optics and opens the door to 
low-cost disposable devices. Its performance was successfully demonstrated in the quantitative measurement of total protein in urine. The photodiode responsivity is $224 \mathrm{~mA} / \mathrm{W}$ with a FWHM of $10 \mathrm{~nm}$ at $\lambda=600 \mathrm{~nm}$. The photodiode sensitivity was $1 \mathrm{kHz} / \mathrm{Wm}^{-2}$ at $\lambda=670 \mathrm{~nm}$.

\section{ACKNOWLEDGMENTS}

The authors which to acknowledge Peter Turmezei, from the Laboratory for Electronic Instrumentation, TUDelft, The Netherlands, for his help with the SU-8 processing. This work is supported by the Portuguese Foundation of Science and Technology (FCT projects SFRH/BD/ 1281/2000 and POCTI/33747/ESE/1999) and by FEDER.

\section{REFERENCES}

[1] P. Connolly, "Clinical diagnostics opportunities for biosensors and bioelectronics," Biosensors \& Bioelectronics, vol. 10, pp. 1-6, 1995.
[2] S. K. Strasinger, M. S. Di Lorenzo, Urianalysis and Body Fluids, $4^{\text {th }}$ ed., F. A. Davis Company, Philadelphia, PA (2001).

[3] http://www.hypoguard.com/diascreen_reagent_strips.h $\mathrm{tml}$.

[4] B. Fowler, A. El Gamal and D. X. D. Yang, "A CMOS area image sensor with pixel-level A/D conversion," ISSCC Digest of Technical Papers, San Francisco, CA, February 1994.

[5] G. de Graaf, R.F. Wolffenbuttel, "Optical CMOS sensor system for detection of light sources," Sensors and Actuators A, vol. 110, pp. 77-81, 2004.

[6] G. Minas, J.C. Ribeiro, R.F. Wolffenbuttel, J.H. Correia, "2-D Modeling and Simulation of Fluidic Microsystems for Biological Fluids Analysis," Proc. MME 2003, Delft, The Netherlands, pp. 239-485, November 2-4, 2003.

[7] SU-8: A Thin Photo-Resister for MEM's, http://aveclafaux.freeservers.com/SU-8.html. 